\title{
Elution Characteristics of Vancomycin, Gentamicin, and Vancomycin/Gentamicin Combination from Calcium Phosphate Cement
}

\author{
Masataka Uchino, ${ }^{1}$ Ken Sugo, ${ }^{2}$ Kouji Naruse, ${ }^{1}$ Kentaro Uchida, ${ }^{3}$ Noriko Hirakawa, \\ Masahiro Toyama, ${ }^{1}$ Genyou Miyajima, ${ }^{1}$ and Ken Urabe ${ }^{1}$ \\ ${ }^{1}$ Department of Orthopaedic Surgery, Kitasato University Medical Center, 6-100 Arai, Kitamoto, Saitama 364-8501, Japan \\ ${ }^{2}$ HOYA Technosurgical Corporation, 1-1-110 Tsutsujigaoka, Akishima, Tokyo 196-0012, Japan \\ ${ }^{3}$ Department of Orthopaedic Surgery, Kitasato University School of Medicine, 1-15-1 Kitasato, Minami-ku, \\ Sagamihara, Kanagawa 252-0375, Japan
}

Correspondence should be addressed to Ken Urabe; kenurabe@med.kitasato-u.ac.jp

Received 11 February 2015; Revised 6 May 2015; Accepted 6 May 2015

Academic Editor: Werner Kolb

Copyright (C) 2015 Masataka Uchino et al. This is an open access article distributed under the Creative Commons Attribution License, which permits unrestricted use, distribution, and reproduction in any medium, provided the original work is properly cited.

\begin{abstract}
The antibiotic elution profiles from calcium phosphate cement (CPC) used for treating infection sites after total joint arthroplasty vary depending on the type and number of impregnated antibiotics. The purpose of this study was to develop a method for efficiently eluting vancomycin hydrochloride (VCM) and gentamicin sulfate (GM) from CPC. Examination of the antibiotic elution profiles of CPC impregnated with either VCM (CPC/V) or GM (CPC/G) or both (CPC/VG) revealed that the early elution of VCM from $\mathrm{CPC} / \mathrm{VG}$ was impaired compared to CPC/V. However, the elution of GM from CPC/VG was similar or higher compared to CPC/G. Scanning electron microscopy showed that the pore structure of CPC markedly differed depending on the type and number of antibiotics present. The pore size of CPC/VG was smaller compared to CPC/V but was larger compared to CPC/G. Thus, the inhibition of the early elution of VCM, which is a larger molecule than GM, was attributed to the decreased pore size of CPC/VG. These findings suggest that when dual treatment with VCM and GM is required for infection following total joint arthroplasty, each antibiotic should be individually impregnated into CPC to maximize the elution efficiency of VCM.
\end{abstract}

\section{Introduction}

Periprosthetic joint infection after total joint arthroplasty is a serious complication that requires prompt treatment. The two-stage exchange procedure is as an effective treatment option for such infections. The first stage of the procedure involves removal of the prosthetic components and bone cement, debridement of necrotic and granulation tissues, and insertion of an antibiotic-impregnated cement spacer. After treatment with intravenous antibiotics, revision total joint arthroplasty is performed in the second stage. The use of antibiotic-impregnated cement spacers is considered to be the optimal method for the localized delivery of antibiotics. However, it has been shown that a treatment failure rate for patients with methicillin-resistant $S$. aureus (MRSA) or methicillin-resistant S. epidermidis (MRSE) infection is higher than the reported rate including patients infected with both nonresistant and resistant organisms [1]. The prevalence of infection caused by MRSA and MRSE has increased in orthopedic patients [2]. Better localized delivery system of antibiotics should be considered for the treatment of MRSA and MRSE.

Recent studies have demonstrated that antibioticsimpregnated calcium phosphate cement (CPC) releases large amounts of antibiotics over long periods [3-5]. We previously compared the elution profiles of vancomycin hydrochloride (VCM) from CPC and PMMA in vitro and found that 8.1-fold more VCM was eluted from CPC than from PMMA in the first $24 \mathrm{~h}$ [6]. Therefore our institution uses both antibiotics-impregnated PMMA and CPC in the first stage 


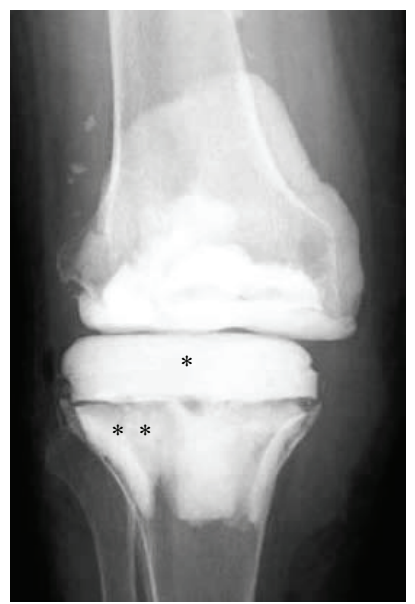

Figure 1: Combination use of antibiotics-impregnated PMMA and $\mathrm{CPC}$ in the first stage of the two-stage exchange procedure in our institution. $*$ : PMMA. $* *$ : CPC.

of the two-stage exchange procedure for the treatment of periprosthetic joint infection as shown in Figure 1.

In the case that the causative microorganism is not detected although physical, hematological, and radiological findings show periprosthetic joint infection, administration of some types of antibiotics should be considered to kill both nonresistant and resistant organisms. In the present study, we confirmed that two types of antibiotics showed the different elution patterns from CPC when each antibiotic was impregnated into CPC separately. We examined whether CPC impregnated with two types of antibiotics with two different elution patterns showed the same elution profiles of each antibiotic to CPC impregnated with each antibiotic alone. Based on these findings, we established a methodology to simultaneously release two types of antibiotics from CPC with similar elution patterns to antibiotics impregnated in CPC alone.

\section{Materials and Methods}

2.1. Materials. CPC (Biopex-R Advance) was obtained from HOYA Technosurgical Corp. (Tokyo, Japan). Injectable VCM and gentamicin sulfate (GM) were purchased from Shionogi (Osaka, Japan) and Nichi-Iko Pharmaceutical (Toyama, Japan), respectively. All other chemicals were obtained from Wako Pure Chemical Industries (Osaka, Japan).

2.2. Preparation of Test Specimens for Double Antibiotic Therapy. VCM and GM were impregnated into CPC to prepare test specimens in triplicate. Briefly, CPC powder (12 g), VCM $(0.3 \mathrm{~g}), 40 \mathrm{mg} / \mathrm{mL} \mathrm{GM}(2.0 \mathrm{~mL})$, and solvent $(0.8 \mathrm{~mL})$ were uniformly mixed to make a paste, which was then applied to a truncated cone-shape silicone mold. The paste was hardened by incubation for $3 \mathrm{~h}$ in a chamber at $37^{\circ} \mathrm{C}$ and $95 \%$ relative humidity. Once hardened, the molds were removed to obtain the test specimens, which were designated as CPC/VG. In addition, reference specimens of CPC impregnated with either VCM or GM, designated as $\mathrm{CPC} / \mathrm{V}$ and $\mathrm{CPC} / \mathrm{G}$, were

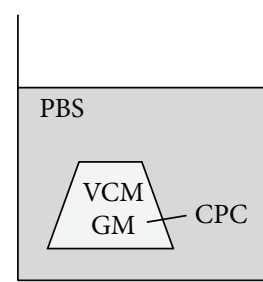

$\mathrm{CPC} / \mathrm{VG}$

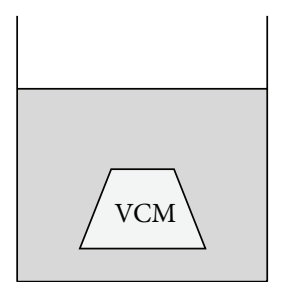

$\mathrm{CPC} / \mathrm{V}$

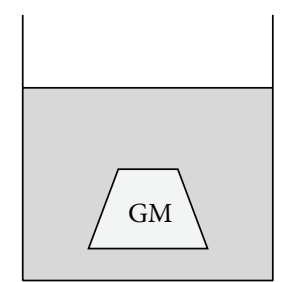

$\mathrm{CPC} / \mathrm{G}$
FIGURE 2: Schematic illustration of the protocol used to measure the elution of VCM and GM from the three types of antibioticimpregnated CPC specimens.

prepared in the same manner as for CPC/VG, but without the addition of GM or VCM, respectively. For CPC/V, CPC powder $(12 \mathrm{~g})$, VCM $(0.3 \mathrm{~g})$, and solvent $(2.8 \mathrm{~mL})$ were mixed into a paste and treated as above. To prepare CPC/G, CPC powder $(12 \mathrm{~g})$, GM $(2.0 \mathrm{~mL})$, and solvent $(0.8 \mathrm{~mL})$ were used.

2.3. Antibiotic Elution. The test and reference specimens of impregnated CPC (1g) were immersed in $10 \mathrm{~mL}$ sterile phosphate-buffered saline (PBS(-)) (Figure 2) and were then incubated at $37^{\circ} \mathrm{C}$ for 16 weeks. PBS(-) was replaced daily. Eluates were collected on days 1, 3, 7, and 14 and weeks 4, 8, 12 , and 16. VCM and GM were detected by high-performance liquid chromatography (HPLC) on the day of collection.

2.4. Determination of VCM Concentration. A series of standard solutions of differing known concentrations of VCM in PBS(-) were prepared and injected into a CAPCELLPAK C18 UG120 column $(5 \mu \mathrm{m}, \varphi 4.6 \mathrm{~mm} \times 25 \mathrm{~cm}$; Shiseido, Tokyo, Japan) of an Elite LaChrom HPLC system (Hitachi High-Technologies, Tokyo, Japan) equipped with a L-2455 Diode Array Detector (Hitachi High-Technologies). The HPLC conditions were as follows: column temperature $30^{\circ} \mathrm{C}$; mobile phase A, triethylamine buffer ( $\mathrm{pH} 3.2$ )/acetonitrile/tetrahydrofuran $=92 / 7 / 1(\mathrm{v} / \mathrm{v})$; isocratic elution, phase A $(20 \mathrm{~min})$; flow rate, $1 \mathrm{~mL} / \mathrm{min}$; wavelength, $280 \mathrm{~nm}$; and injection volume, $20 \mu \mathrm{L}$. The peak area of VCM in each standard solution was measured and plotted against the VCM concentration to generate a calibration curve. The concentration of VCM in each eluate sample was then determined by HPLC under the same conditions using the standard calibration curve.

2.5. Determination of GM Concentration. A series of standard solutions of differing known concentrations of GM in PBS(-) separately are injected into a CAPCELL-PAK C18 UG120 column $(5 \mu \mathrm{m}, \varphi 4.6 \mathrm{~mm} \times 25 \mathrm{~cm})$ of an Elite LaChrom HPLC system (Hitachi High-Technologies) equipped with a L-2480 Fluorescence Detector (Hitachi High-Technologies). The three peak areas, which corresponded to the $\mathrm{Cl}, \mathrm{Cla}$, and C2 components of GM, in each standard GM solution were measured and plotted against the GM concentration to generate a calibration curve. The concentration of GM in each eluate sample was then determined by HPLC using the standard calibration curve. As GM has no specific UV absorption, 
postcolumn derivatization with o-phthalaldehyde was used to form fluorescent products for the determination of GM concentration, according to a method described by Anhalt [7].

2.6. Pore Structure and Porosimetry Analyses. The relationship between the pore structure and antibiotic elution profiles of the test and reference specimens was analyzed and compared. To prepare CPC/VG, CPC powder $(3 \mathrm{~g})$, VCM $(0.075 \mathrm{~g}), \mathrm{GM}(0.5 \mathrm{~mL})$, and solvent $(0.2 \mathrm{~mL})$ were mixed uniformly to prepare a paste, which was then placed in a cylindrical acrylic mold and hardened by incubation for $3 \mathrm{~h}$ at $37^{\circ} \mathrm{C}$ and $95 \%$ relative humidity. The molds were removed to obtain the specimens. $\mathrm{CPC} / \mathrm{V}$ and $\mathrm{CPC} / \mathrm{G}$ test specimens were prepared in the same manner as that used for $\mathrm{CPC} / \mathrm{VG}$. CPC/V was prepared using $\mathrm{CPC}$ powder $(3 \mathrm{~g})$, VCM $(0.075 \mathrm{~g})$, and solvent $(0.7 \mathrm{~mL})$, and $\mathrm{CPC} / \mathrm{G}$ was prepared using CPC powder $(3 \mathrm{~g}), \mathrm{GM}(0.5 \mathrm{~mL})$, and solvent $(0.2 \mathrm{~mL})$. The obtained specimens were immersed in $20 \mathrm{~mL}$ PBS(-) and incubated at $37^{\circ} \mathrm{C}$ for $24 \mathrm{~h}$. The PBS(-) was then replaced with $20 \mathrm{~mL}$ acetone for dehydration of the specimens. After $10 \mathrm{~min}$, the specimens were removed from the acetone and then dried at room temperature. Thin sections (1-2 mm thickness) of each specimen were prepared using a microtome for scanning electron microscopy (SEM) analysis (S-4300, Hitachi High-Technologies) and the analysis of pore size using a mercury porosimetry (AutoPore IV9520, Micromeritics, Norcross, GA, USA).

\section{Results and Discussion}

3.1. VCM Elution from CPC. The elution of VCM from CPC was monitored by measuring the concentration of the released antibiotic over a 16 -week period (Figure 3 ). The elution continued for several weeks, although the concentration gradually decreased after reaching a peak at $24 \mathrm{~h}$. The elution of VCM during the first 7 days was reduced by approximately two-thirds from CPC impregnated with both VCM and GM (CPC/VG) compared to CPC/V. As the elution of VCM from CPC/VG was clearly inhibited, the target MIC and associated antibacterial effect would not be achieved with this material at sites of periprosthetic joint infection. All tests were run in triplicate and averaged. A triplicate test is enough, because, in such experiment system, there are few errors so that error bars in the figure hide in the closed circles.

3.2. GM Elution from CPC. The time course for the elution of GM from CPC/G and CPC/VG was also examined (Figure 4). In contrast to the elution profile of VCM, that of GM was nearly identical from both single- and double-impregnated CPC throughout the measurement period. As shown in Figures 3 and 4, approximately 4-fold higher amounts of VCM were eluted from CPC compared to GM. All tests were run in triplicate and averaged. A triplicate test is enough, because, in such experiment system, there are few errors so that error bars in the figure hide in the closed circles.

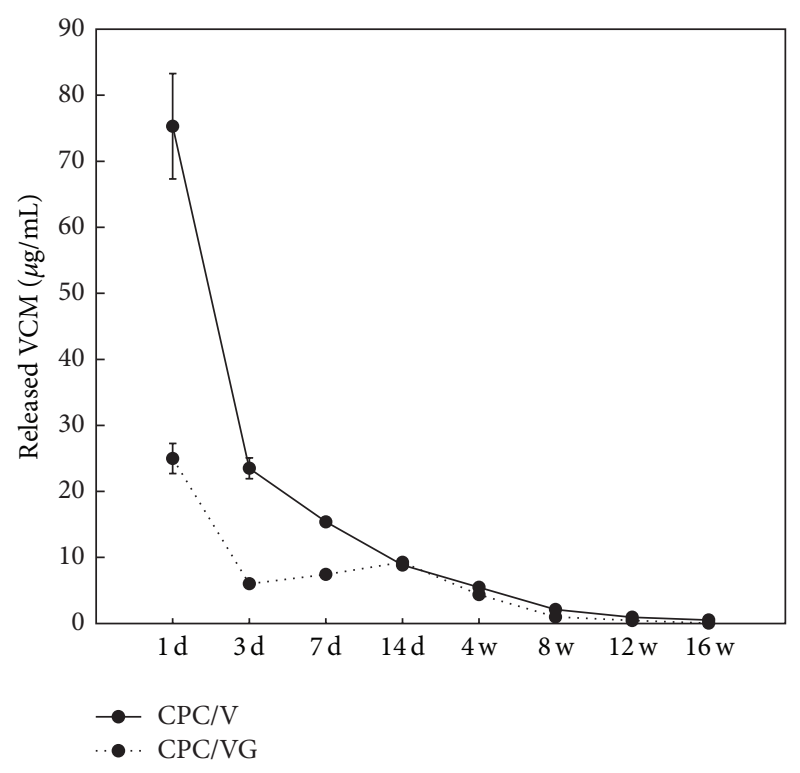

FIGURE 3: Elution of VCM from CPC/V and CPC/VG. Measurements were performed on the indicated days/weeks. Data are presented as the mean \pm standard deviation of triplicate experiments.

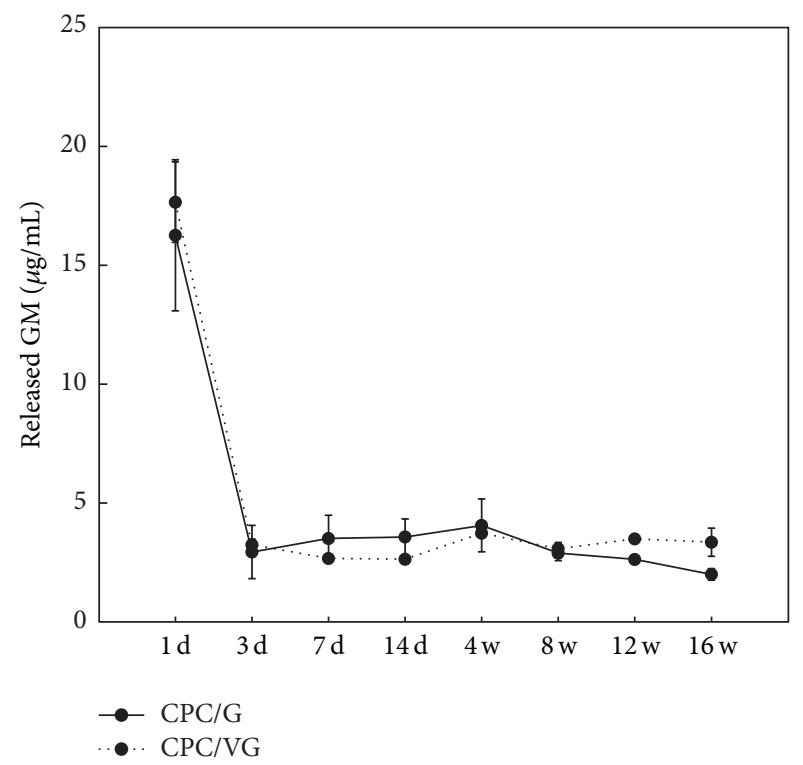

FIGURE 4: Elution of GM from CPC/G and CPC/VG. Measurements were performed on the indicated days/weeks. Data are presented as the mean \pm standard deviation of triplicate experiments.

3.3. SEM Observation. Innumerable pores are present on the surface and within CPC and act as passages for antibiotic elution [8]. To investigate why the elution patterns of VCM and GM from CPC differed depending on the type and number of impregnated antibiotics, we therefore analyzed the pore structure of the various antibiotic-impregnated CPC specimens by SEM. The SEM images differed markedly between the $\mathrm{CPC} / \mathrm{V}, \mathrm{CPC} / \mathrm{G}$, and $\mathrm{CPC} / \mathrm{VG}$ specimens (Figure 5). The surface and interior of $\mathrm{CPC} / \mathrm{V}$ were comprised of 


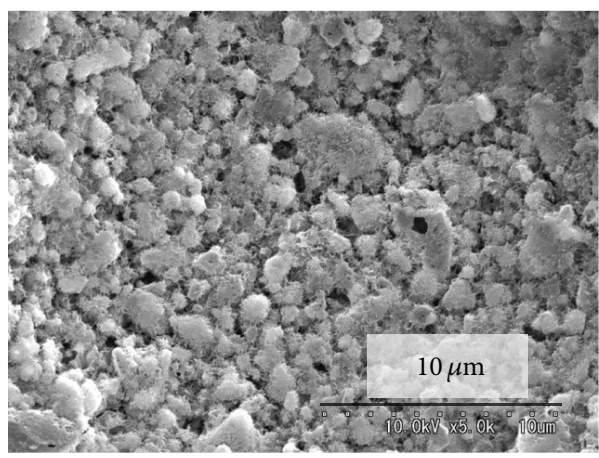

(a)

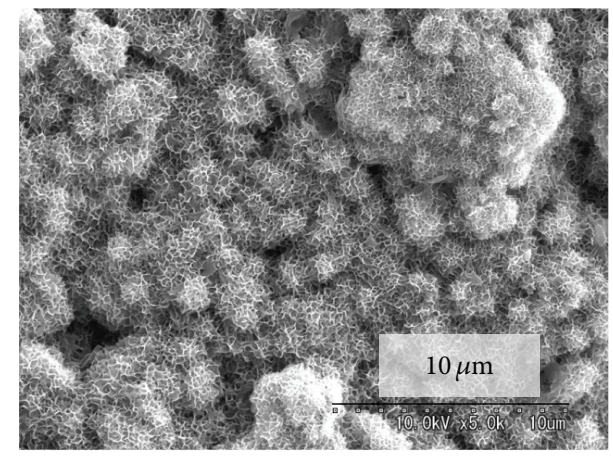

(b)

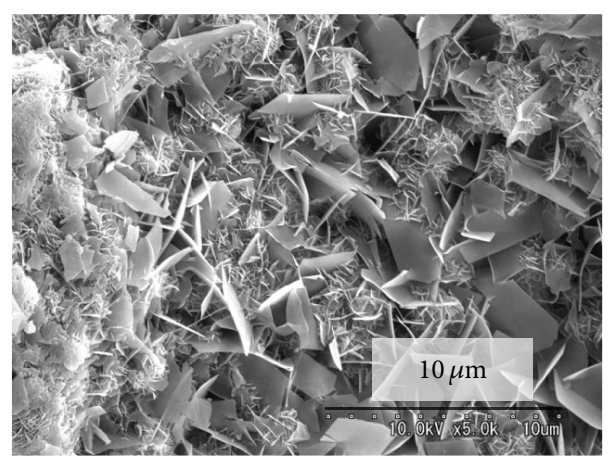

(c)

Figure 5: SEM images of the surfaces of CPC/V (a), CPC/VG (b), and CPC/G (c).

cobblestone-like microstructures, whereas $\mathrm{CPC} / \mathrm{G}$ contained numerous needle-like projections. Interestingly, $\mathrm{CPC} / \mathrm{VG}$ appeared to have a structure that combined the $\mathrm{CPC} / \mathrm{V}$ and $\mathrm{CPC} / \mathrm{G}$ characteristics, in which the cobblestone-like microstructures were covered in smaller needle-like projections.

3.4. Porosimetry Analysis. Mercury porosimetry was used to measure the pore size distribution of the antibioticimpregnated CPC specimens (Figure 6). Consistent with the SEM analysis, the pore size was found to change depending on the type and number of impregnated antibiotics. Specifically, impregnation with VCM increased the pore size of CPC, whereas GM decreased the pore size of CPC. In CPC/VG, the average pore was intermediate in size between the larger pores observed in $\mathrm{CPC} / \mathrm{V}$ and the smaller pores found in $\mathrm{CPC} / \mathrm{G}$. The results of the porosimetry analysis suggest that the reduced elution of VCM from CPC impregnated with VCM and GM under double antibiotic therapy conditions was due to the decrease in CPC pore size. Conversely, based on the increase in $\mathrm{CPC} / \mathrm{G}$ pore size after impregnation with $\mathrm{VCM}$, the elution of GM would be expected to be enhanced from CPC/VG; however, as demonstrated from the GM elution profile presented in Figure 4, the amount of GM in the eluate was not markedly affected. The differential effects on the elution of VCM and GM in response to the change in pore size may be explained by the sizes of these antibiotics. Compared with VCM, which has a MW of approximately 1450, the MW of GM is only 480. Thus, the increase in pore size resulting from the introduction

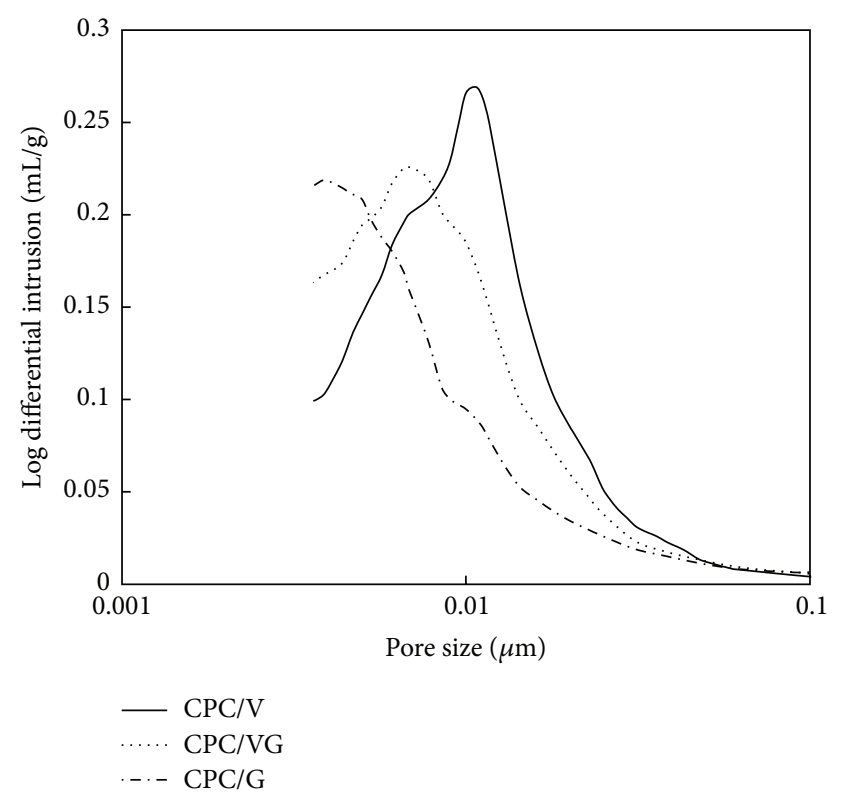

FIGURE 6: Results of mercury porosimetry analysis for $\mathrm{CPC} / \mathrm{V}$, $\mathrm{CPC} / \mathrm{G}$, and $\mathrm{CPC} / \mathrm{VG}$.

of VCM into CPC/G would not be expected to markedly affect the elution of the relatively small GM, whereas the decrease in pore size resulting from the introduction of GM into CPC/V appears to have markedly decreased the elution of the larger VCM. This result is the problem of pore size 


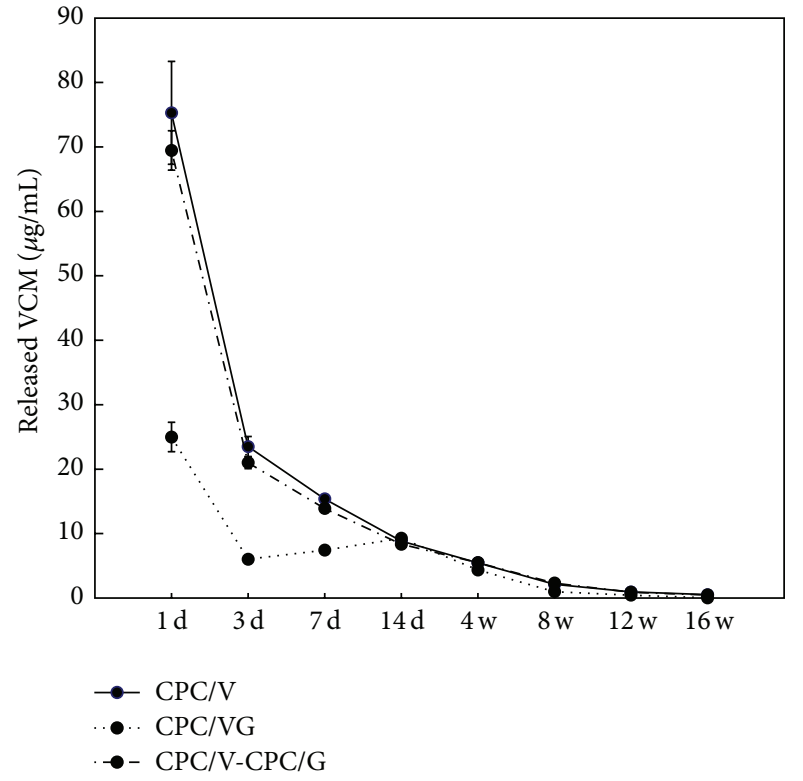

FIGURE 7: Elution profiles of VCM from CPC/V, CPC/VG, and $\mathrm{CPC} / \mathrm{V}-\mathrm{CPC} / \mathrm{G}$. Measurements were performed on the indicated days/weeks. Data are presented as the mean \pm standard deviation of triplicate experiments.

and not an antagonistic interaction between VCM and GM, according to another preliminary experiment. It indicated that the residual titer of VCM $(0.3 \mathrm{~g})$, after mixing with $2.0 \mathrm{~mL}$ of GM $(40 \mathrm{mg} / \mathrm{mL})$, showed $99.7 \%\left(37^{\circ} \mathrm{C}, 24 \mathrm{~h}\right)$. The reduction of VCM, as described in this paper, could not be observed only by combining both antibiotics.

3.5. Improved Antibiotic Elution System. As the above results demonstrated that the elution of VCM from CPC was markedly reduced when both GM and VCM were impregnated into $\mathrm{CPC}$, we attempted to increase the elution of VCM by using single antibiotic-impregnated CPC specimens, $\mathrm{CPC} / \mathrm{V}$ and $\mathrm{CPC} / \mathrm{G}$, immersed together in PBS(-), designated as $\mathrm{CPC} / \mathrm{V}-\mathrm{CPC} / \mathrm{G}$. The elution profile of $\mathrm{VCM}$ from $\mathrm{CPC} / \mathrm{V}$ located in close proximity to $\mathrm{CPC} / \mathrm{G}$ was monitored over a 16 -week period (Figure 7). As can be seen from the VCM elution curve, the elution profiles of VCM from $\mathrm{CPC} / \mathrm{V}$ and $\mathrm{CPC} / \mathrm{V}-\mathrm{CPC} / \mathrm{G}$ were nearly identical, whereas that from $\mathrm{CPC} / \mathrm{VG}$ was comparatively reduced by approximately twothirds. Therefore, the inhibition of VCM elution from CPC was clearly alleviated using this approach. All tests were run in triplicate and averaged. A triplicate test is enough, because, in such experiment system, there are few errors so that error bars in the figure hide in the closed circles.

\section{Conclusions}

The present findings demonstrate that the impregnation of CPC with both VCM and GM inhibits the early elution of VCM when compared to CPC impregnated with VCM only. Notably, the pore size of CPC impregnated with VCM and GM was smaller than that found in $\mathrm{CPC} / \mathrm{V}$, suggesting that this decrease in pore size impaired the elution of the relatively large VCM molecule. When dual antibiotic treatment with VCM and GM is used for infection after total joint arthroplasty, CPC impregnated with VCM and GM individually should be used.

\section{Conflict of Interests}

The authors declare that there is no conflict of interests regarding the publication of this paper.

\section{References}

[1] F. Leung, C. J. Richards, D. S. Garbuz, B. A. Masri, and C. P. Duncan, "Two-stage total hip arthroplasty: how often does it control methicillin-resistant infection?" Clinical Orthopaedics and Related Research, vol. 469, no. 4, pp. 1009-1015, 2011.

[2] W. E. Shams and R. P. Rapp, "Methicillin-resistant staphylococcal infections: an important consideration for orthopedic surgeons," Orthopedics, vol. 27, no. 6, pp. 565-568, 2004.

[3] O. Kisanuki, H. Yajima, T. Umeda, and Y. Takakura, "Experimental study of calcium phosphate cement impregnated with dideoxy-kanamycin B," Journal of Orthopaedic Science, vol. 12, no. 3, pp. 281-288, 2007.

[4] T. Sasaki, Y. Ishibashi, H. Katano, A. Nagumo, and S. Toh, "In vitro elution of vancomycin from calcium phosphate cement," The Journal of Arthroplasty, vol. 20, no. 8, pp. 1055-1059, 2005.

[5] M. Suzuki, T. Tsukeoka, Y. Tsuneizumi et al., "Mechanical strength and in vitro antibiotic release profile of antibioticsloaded calcium phosphate bone paste," Clinical Orthopaedic Surgery, vol. 39, no. 3, pp. 309-314, 2004 (Japanese).

[6] K. Urabe, K. Naruse, H. Hattori et al., "In vitro comparison of elution characteristics of vancomycin from calcium phosphate cement and polymethylmethacrylate," Journal of Orthopaedic Science, vol. 14, no. 6, pp. 784-793, 2009.

[7] J. P. Anhalt, "Assay of gentamicin in serum by high pressure liquid chromatography," Antimicrobial Agents and Chemotherapy, vol. 11, no. 4, pp. 651-655, 1977.

[8] S. Sugiyama, M. Itokazu, T. Akaike, Y. Nonomura, and K. Shimizu, "Drug delivery system for antibiotics using calcium phosphate cement," Orthopedic Surgery, vol. 55, no. 3, pp. 357362, 2004 (Japanese). 


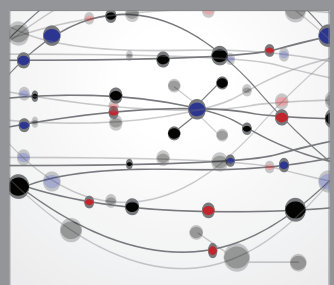

The Scientific World Journal
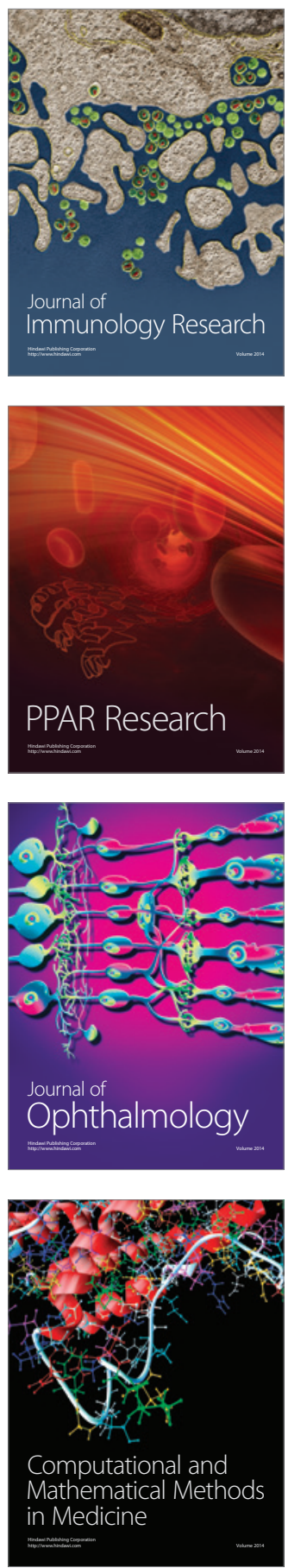

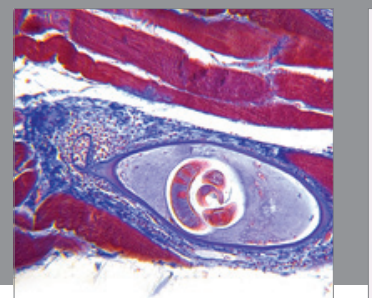

Gastroenterology

Research and Practice
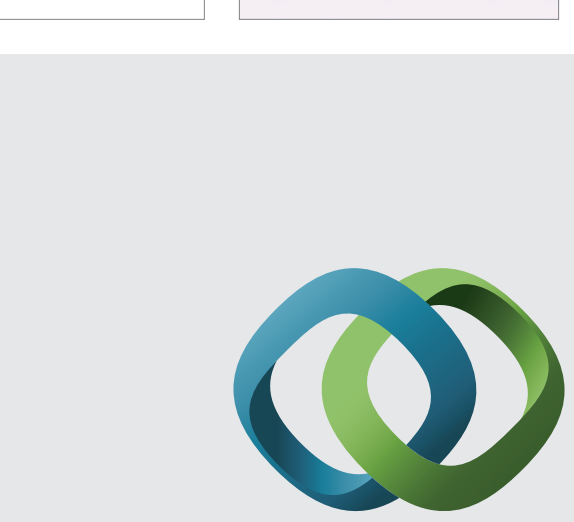

\section{Hindawi}

Submit your manuscripts at

http://www.hindawi.com
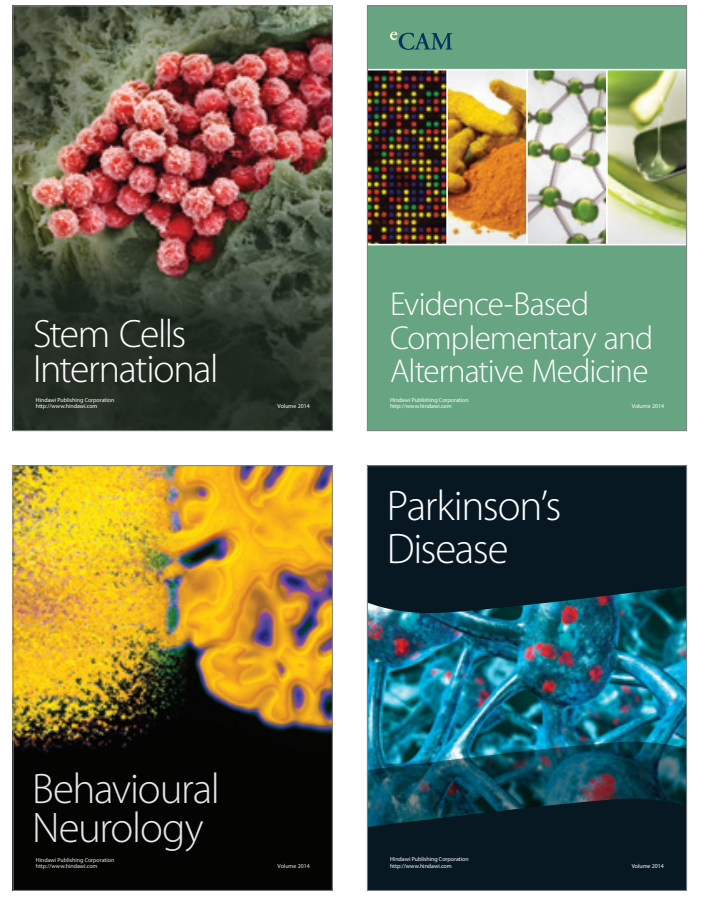
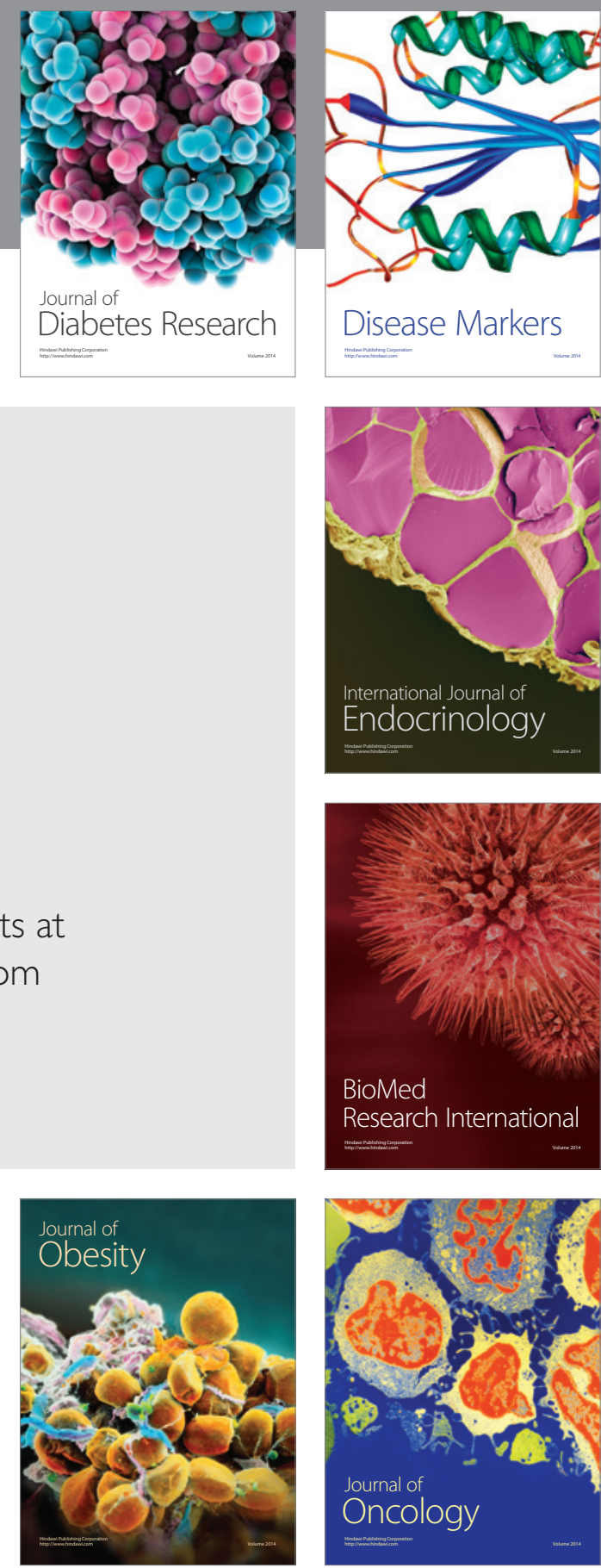

Disease Markers
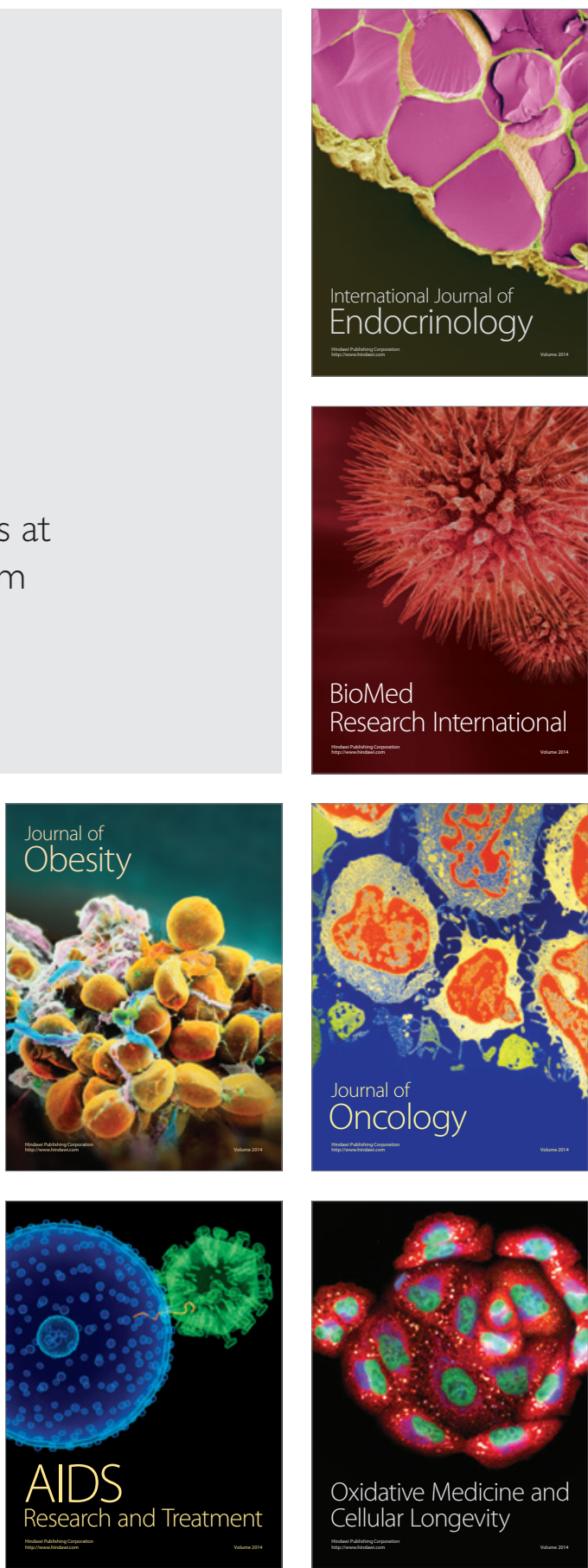